\title{
Investigations of the electronic structure of nanoscaled gold-colloids in sol-gel-coatings
}

\author{
B. Kutsch ${ }^{\text {a. } *}$, O. Lyon ${ }^{\text {b }}$, M. Schmitt ${ }^{\text {a }}$, M. Mennig ${ }^{\text {a }}$, H. Schmidt ${ }^{\text {a }}$ \\ ${ }^{a}$ Institut für Neue Materialien, Im Stadtwald, Gebäude 43, D-66123 Saarbrïcken, Germany \\ ${ }^{\mathrm{b}}$ Laboratoire pour l'Utilisation du Rayonnement Électromagnétique (LURE), Bâtiment 209D, Centre Universitaire Paris-Sud, F-91405 \\ Orsay, France
}

\begin{abstract}
The electronic structure of nano-scaled Au-colloids embedded in thin glass-like sol-gel coatings on glass substrates was investigated by small-angle X-ray scattering (SAXS) in grazing incidence and by UV-VIS spectroscopy as a function of the densification temperature of the coatings and of kind and concentration of the stabilizer used in the sol. Special interest was concentrated on the structure of the interface between matrix and colloids. It was found that the structure of this interface changes with the preparation of the coatings. Depending on densification temperature and stabilizer, it was observed that the structure of the surface of the colloids or the interface region between matrix and colloids is changing continuously from a structure similar to a fractal surface to a sharp phase boundary. Further densification leads partially to a 'fuzzy' colloid surface which is supposed to be due to the formation of a highly densified shell around the colloids caused by the ligands.
\end{abstract}

\section{Introduction}

The investigation of the electronic structure of alloys, glasses, microemulsions and gels by SAXS in order to get information about fluctuations of their electronic density is of great interest in materials research. A review of work of the past years is given in [1-3]. In this paper, interest is concentrated on the electronic structure of thin $(1-2 \mu \mathrm{m})$ glass-like solgel coatings containing nanoscale gold colloids dipcoated on glass substrates. In order to prevent an agglomeration of the gold in the sol before coating

\footnotetext{
* Corresponding author. Tel.: +49-681 302 5092; fax: +49-681 302 5242; e-mail: kutsch@inm.uni-sb.de.
}

and thermal densification, functionalized silanes have been used as stabilizers to complex the gold ions in the sol. Colloids are then formed in situ during densification of the matrix by thermal reduction of complexed gold ions [4,5]. Stabilizers have a great influence on size and size distribution of the colloids within the coatings and were recently found to influence strongly the optical properties of the colloids [6]. The mechanisms of this influence are not well understood yet, but it is supposed that the electronic properties (i.e., conductivity, dielectric properties, electronic density) of the interface between sol-gel matrix and embedded colloids play an important role in these effects. One promising method to get an access to this interface in order to investigate its 
structural properties is SAXS, as it has been demonstrated for example in reference [7-10]. The last article is concerned with the structure of anodically oxidized porous silicon layers summarizes the main ideas in this field.

Due to the low thickness of the coatings, SAXS experiments had to be done in grazing incidence geometry using an angle of incidence $\alpha$ of $0.3^{\circ}$. This leads to an augmentation of the effective $\mathrm{X}$-ray path within the sample by a factor of about 200 . This technique was proposed some years ago by Naudon et al. $[11,12]$. Its feasibility for application at thin sol-gel coatings containing Au colloids was proved in a previous paper [13] where its results were compared with electron microscopy and UV-VIS spectroscopy. Theoretical aspects and data correction procedures applied for GISAXS (grazing incidence SAXS) as well as details of the experimental set-up are given there.

\section{Experimental procedure}

\subsection{Instrumentation}

Experiments were performed at the beam line D22 at LURE (Laboratoire pour l'Utilisation du Rayonnement Électromagnétique) [14]. Details of the experimental set-up are given in [13] so that explanations can be restricted to a short survey. The sample holder used for the experiments in grazing incidence is sketched in Fig. 1. It could be rotated in an arbitrary way to modify $\alpha$ and shifted in height by $\pm 7 \mathrm{~mm}$. The incident $X$-ray beam was collimated to

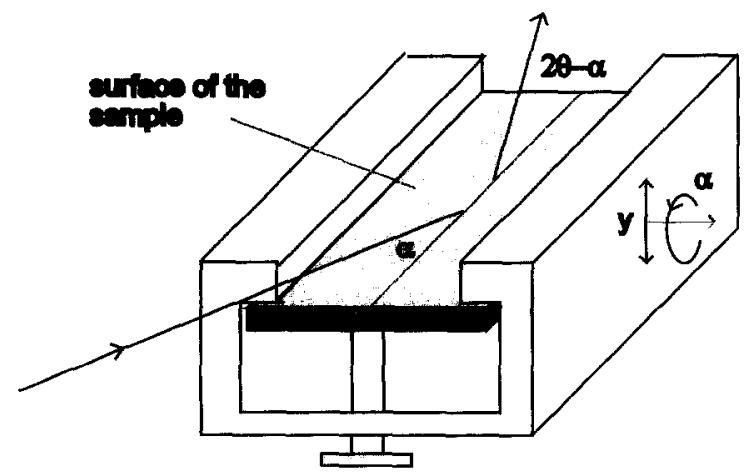

Fig. 1. Schematic representation of the rotatable sample holder used for experiments in grazing incidence. a height of $100 \mu \mathrm{m}$ and a width of $1 \mathrm{~mm}$. As X-ray detector, a two-dimensional position-sensitive proportional counter with a $\mathrm{Xe}-\mathrm{CH}_{4}$-mixture was used. It had 256 channels in each direction and a space resolution of $0.334 \mathrm{~mm} \times 0.334 \mathrm{~mm}$. The sample to detector distance was $657 \mathrm{~mm}$. With $\alpha=0.3^{\circ}$ the experimental limit of the observable radii of the colloids can be estimated to be about $1.0 \mathrm{~nm}$ to 10 $\mathrm{nm}$ at $8050 \mathrm{eV}$. To avoid a strong total reflection of the incident X-ray beam, $\alpha$ was selected as $0.3^{\circ}$. The remaining total reflection was masked with an additional vertical beamstop. The scattering of the colloids could be clearly identified by a variation of the X-ray energy (ASAXS) at the $\mathrm{L}_{\mathrm{III}}$-absorption edge of gold (11919 eV) [13].

Scattering of the pure matrix was neglectible compared with samples containing Au-colloids. UVVIS measurements have been done with a Bruins Omega 20. Coating thickness was determinated by ellipsometry using a ES4G spectrometer of SOPRA. Additionally, investigations by secondary neutral mass spectroscopy (SNMS) with an INA 3 instrument of Leybold have been done to get the composition of the coatings and to check their thickness. High resolution transmission electron microscopy (HRTEM) was performed with a Philips CM 200 by scraping a splinter of the sol-gel coating containing Au-colloids onto a carbon net.

\subsection{Data correction procedures for SAXS in grazing incidence}

All data have been corrected in two dimensions for absorption and refraction effects as reported in [13], using coating thickness, composition and calculated critical angle of total reflection. All spectra showed a spherical symmetry of the intensity distribution around the origin after these procedures. Thus, they were integrated within concentric circles to get one-dimensional spectra after the corrections. All the spectra were finally corrected for detector efficiency versus energy and position as well as for incident photon flux and background signal.

\subsection{Description of the samples}

For the preparation of the glass-like sol-gel coatings containing Au colloids, $1.0 \mathrm{~g}$ of $\mathrm{H}\left[\mathrm{AuCl}_{4}\right] \cdot \mathrm{H}_{2} \mathrm{O}$ 


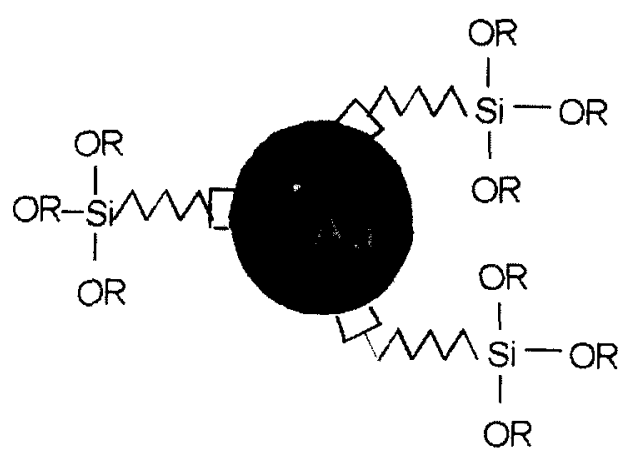

Fig. 2. Schematic model of the stabilization of the nanoscaled gold colloids within the sol-gel coatings by functionalized ligands (DIAMO, THIO) after reduction.

was dissolved in a mixture of $15 \mathrm{ml}$ ethanol. Then $\mathrm{N}$-(2-aminoethyl)-3-aminopropyltrimethoxysilane (DIAMO) or 3-mercaptopropyltrimethoxysilane (THIO) were added to the solution. In this work samples will be discussed with molar ratios of Au:DIAMO = 1:1, 1:2 and 1:4 as well as Au:THIO $=1: 1$. Finally $18.8 \mathrm{ml}$ of the matrix sol, prepared from $160 \mathrm{ml}$ 3-glycidoxypropyltriethoxysilane (GPTS) and $40 \mathrm{ml}$ tetraethylorthosilicate (TEOS), dissolved in $240 \mathrm{ml}$ ethanol and prehydrolyzed with
$28.5 \mathrm{ml}$ of $0.1 \mathrm{M} \mathrm{HNO}_{3}$ was added and stirred for 3-5 min until a clear solution was obtained. After these procedures, soda-lime glass microscopic slides were dip-coated with a withdrawal speed of $10 \mathrm{~mm} / \mathrm{s}$ and dried at $80^{\circ} \mathrm{C}$ for $1 \mathrm{~h}$ at ambient air to remove the solvents from the gel.

Finally, the coatings were further densified under ambient air between $150^{\circ} \mathrm{C}$ and $300^{\circ} \mathrm{C}$ in $50 \mathrm{~K}$ steps for $1 \mathrm{~h}$ (heating rate: $60 \mathrm{~K} / \mathrm{h}$ ). More detailed information about the preparation of the coatings can be found in [5,15] A schematic model to the influence of the stabilizer after reduction of the Au colloids within the sol-gel coatings is presented in Fig. 2, suggesting the influence of the functionalized ligands at the surface of the colloids.

\section{Results}

\subsection{Characterization by UV-VIS spectroscopy}

The UV-VIS absorbance spectra of the coatings as a function of densification temperature and kind and concentration of the stabilizer are presented in

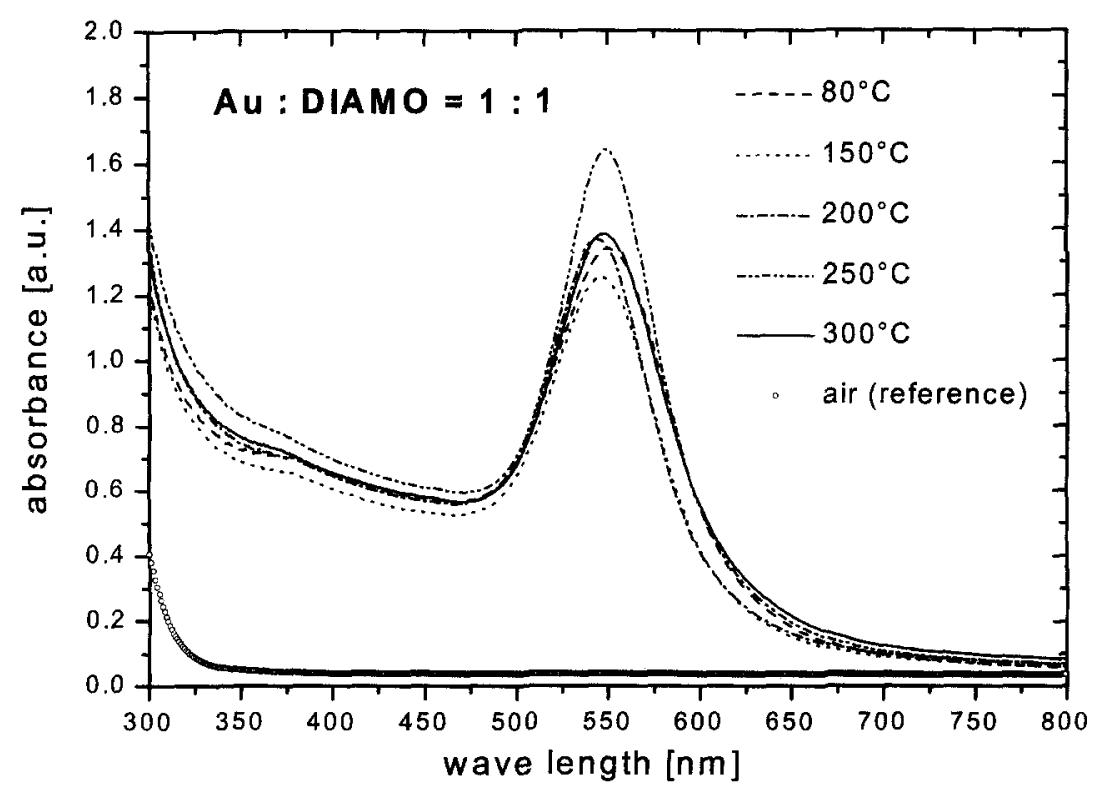

Fig. 3. UV-VIS spectra of the coatings densified between $80^{\circ} \mathrm{C}$ and $300^{\circ} \mathrm{C}$ in air (molar ratio Au:DIAMO = 1:1). 


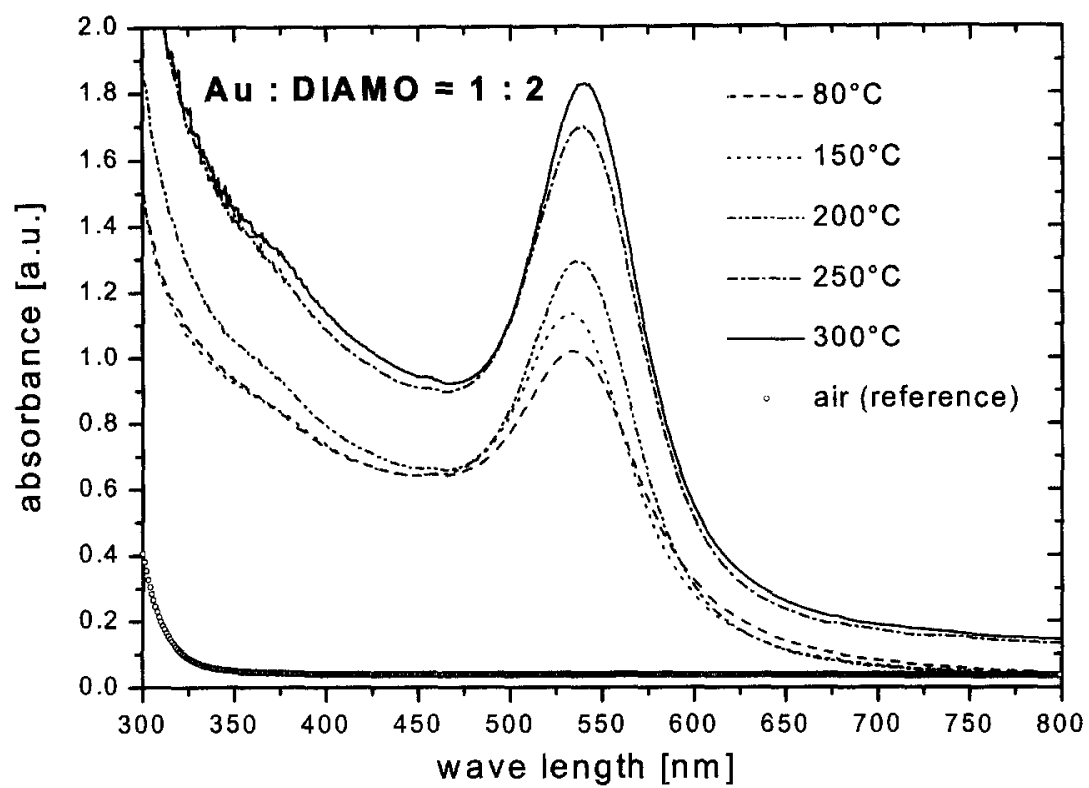

Fig. 4. UV-VIS spectra of the coatings densified between $80^{\circ} \mathrm{C}$ and $300^{\circ} \mathrm{C}$ in air (molar ratio Au:DIAMO $=1: 2$ ).

Figs. 3-6. In the case of DIAMO, all spectra clearly show the expected plasma resonance peak of nanoscaled Au-colloids. Coatings stabilized with
THIO (molar ratio: 1:1) were colorless to temperatures of $150^{\circ} \mathrm{C}$. At $200^{\circ} \mathrm{C}$, a slight absorbance of Au-colloids can be seen which continuously in-

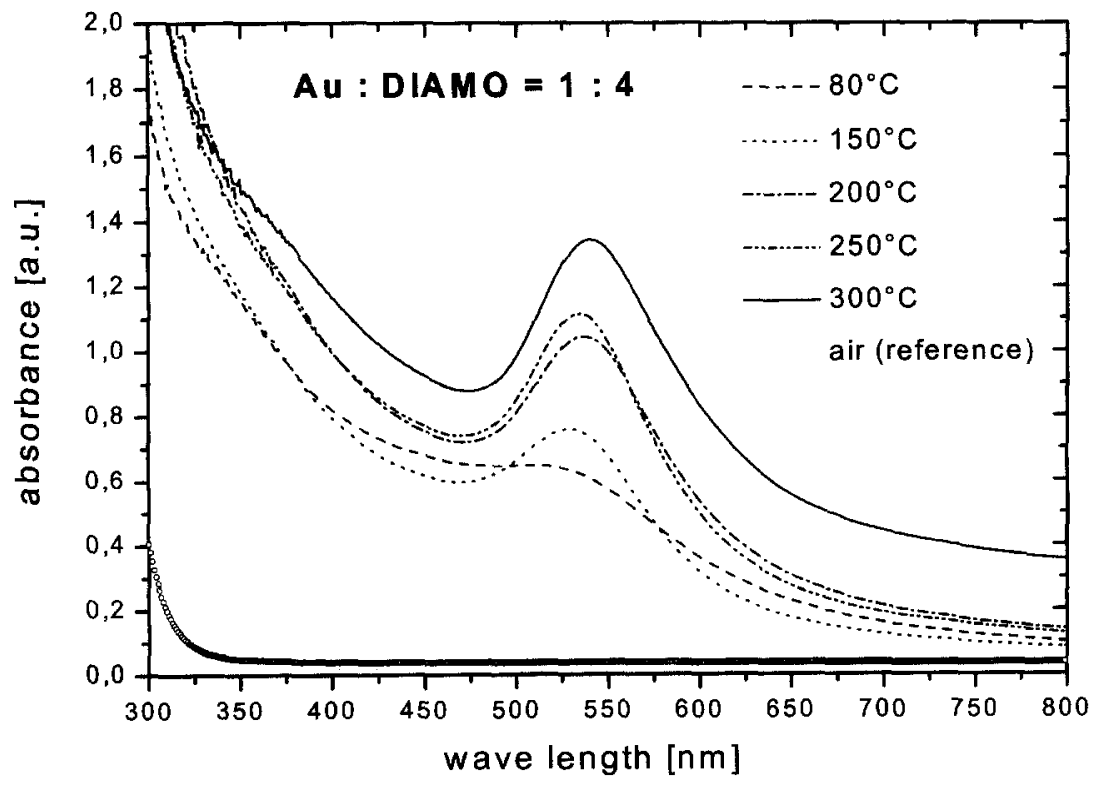

Fig. 5. UV-VIS spectra of the coatings densified between $80^{\circ} \mathrm{C}$ and $500^{\circ} \mathrm{C}$ in air (molar ratio Au:DIAMO $=1: 4$ ). 


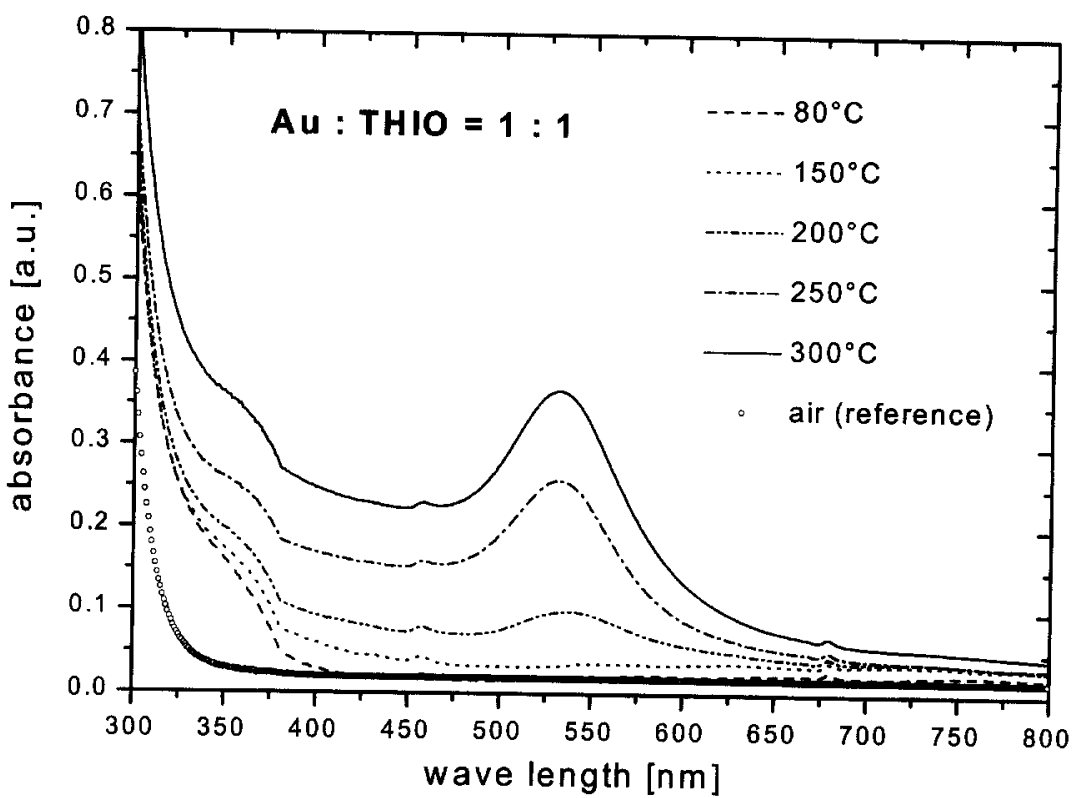

Fig. 6. UV-VIS spectra of the coatings densified between $80^{\circ} \mathrm{C}$ and $300^{\circ} \mathrm{C}$ in air (molar ratio Au:THIO $=1: 1$ ).

creases with densification. The appearance of these Au-colloids is also clearly seen in the corresponding SAXS-spectra (see Fig. 10) and indicates the sensitivity of both methods for small amounts of colloidal gold.

\subsection{SAXS-experiments}

The spectra in Figs. 7-10 show the corrected SAXS spectra $(E=8050 \mathrm{eV})$ in the case of coatings densified at $80^{\circ} \mathrm{C}$ to $300^{\circ} \mathrm{C}$ in grazing incidence for

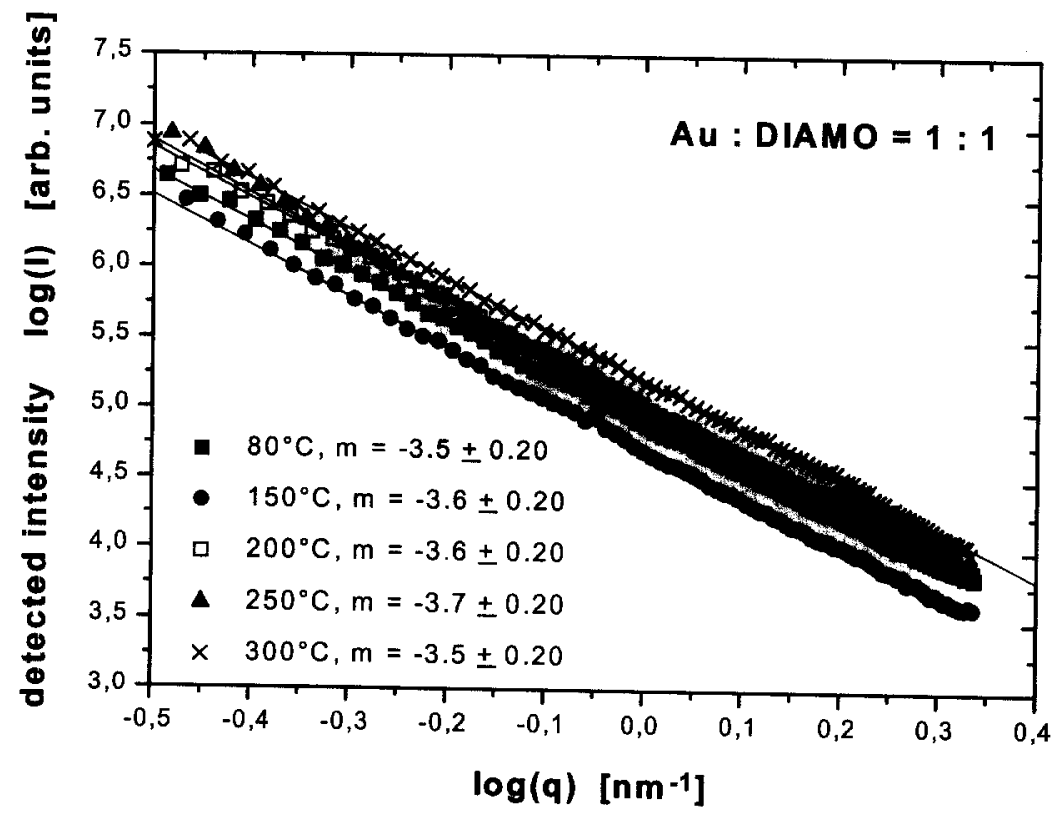

Fig. 7. Scattering spectra in a $\log (I)$ vs. $\log (q)$ representation (Au:DIAMO $=1: 1$ ) as a function of densification. 


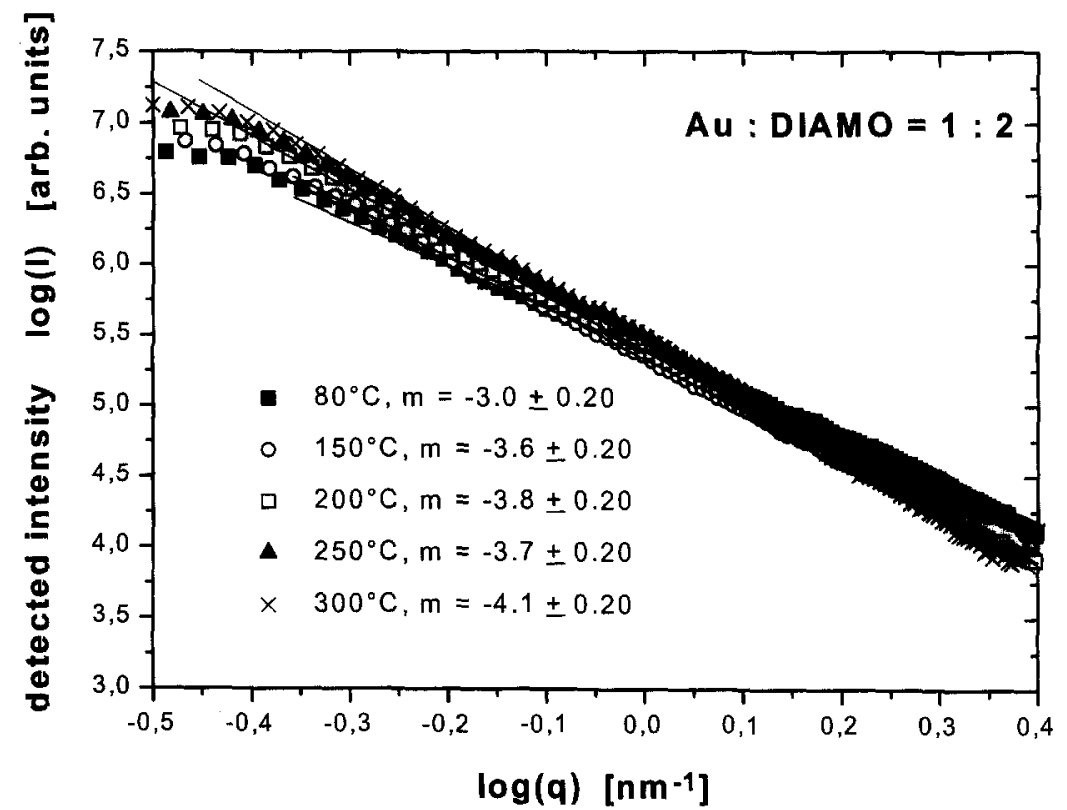

Fig. 8. Scattering spectra in a $\log (I)$ vs. $\log (q)$ representation (Au:DIAMO $=1: 2$ ) as a function of densification.

the stabilizers DIAMO (molar ratios: $1: 1,1: 2$ and $1: 4)$ and THIO (molar ratio: $1: 1)$ in a $\log (I)$ vs. $\log (q)$ representation. These spectra were fitted in their linear range in order to determine the slopes as indicated in the plots within an estimated precision of about +0.25 mainly due to the restricted $q$-range

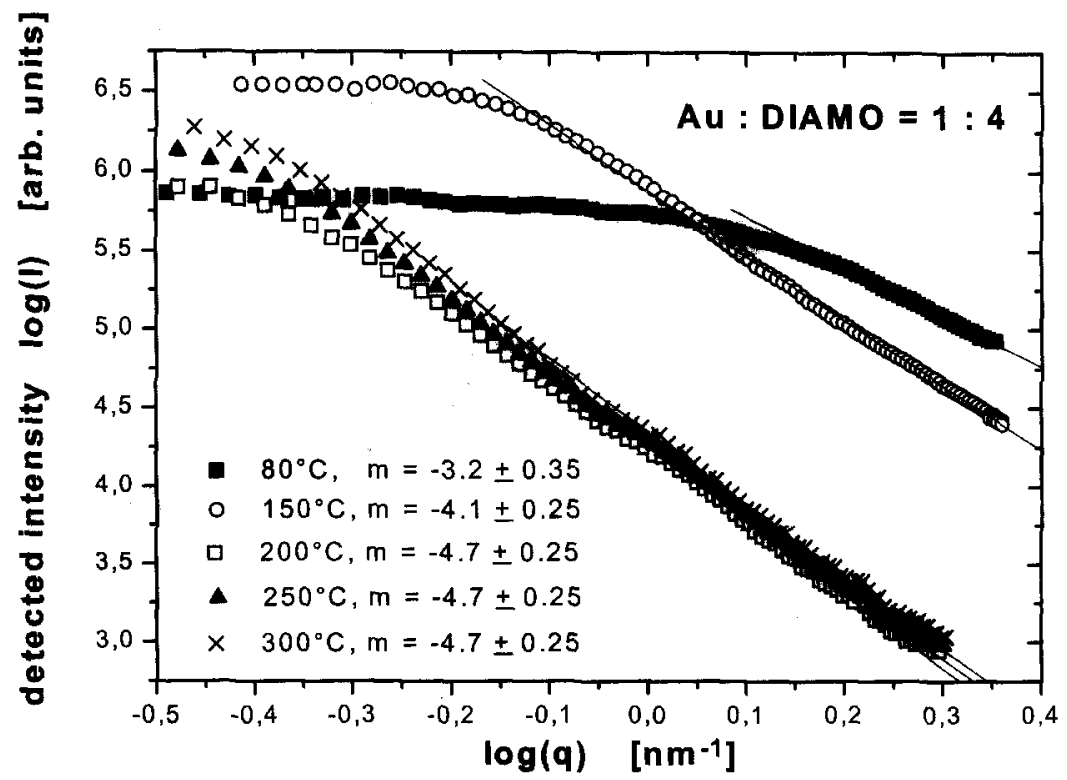

Fig. 9. Scattering spectra in a $\log (I)$ vs. $\log (q)$ representation (Au:DIAMO $=1: 4$ ) as a function of densification. 



\section{Discussion}

A comparison of calculated Mie-spectra and measured UV-VIS spectra should in principle allow determination of mean Au-colloid size. This procedure fails, however, in the case of our samples as the experimental FWHM values are untypical high (generally indicating small colloids, see for example Fig. 5) and cannot be explained even by a wide size distribution. It can only be stated by this method that their size increases temperature on a scale between about 1 to $10 \mathrm{~nm}$. The reason for this behavior which strongly depends on kind and concentration of the stabilizer used in the sol [5] is not well understood yet. It is supposed that the dielectric properties of the interface between matrix and colloids cause these effects $[18,19]$. This problem was one motivation for further investigations by SAXS to get an access to the electronic structure of the interface.

The UV-VIS spectra in Fig. 3 show that in the case of a ratio $\mathrm{Au}: \mathrm{DIAMO}=1: 1$, which is expected not to allow a stabilization of all $\mathrm{Au}$ ions (assuming $\mathrm{Au}^{3+}$ ) within the sol [20], no systematic change of the spectra with temperature can be observed. The formation of the colloids seems to be spontaneous and the amount of colloidal gold does not vary systematically with temperature. A shift of the maximum position of the plasma resonance peak or a change of its FWHM which in general indicates a change of the colloid's size is not detectable. This is in agreement with Table 1 where only erratic changes of the size of the colloids were observed which underline the interpretation of a spontaneous and largely uncontrolled reduction of Au-ions which are not or only weak complexed by the functionalized silanes. Growth of particles with higher densification temperature as it expected assuming an Ostwald ripening mechanism or a diffusion of unreduced Au-ions or atoms into the colloids was not observed. Together with the results from Table 2 it is supposed, thus, that only few unreduced gold-ions in the surrounding of the colloids exist. In fact, a ratio of 1:1 allows neither to control of the size of the colloids, nor the amount of colloidal gold.

At a molar ratio of Au:DIAMO $=1: 2$ (Fig. 4), which should enable the ligands to co-ordinate all Au-ions assuming a square planar complexation (chelate complex) in the sol, a continuous augmenta- tion of the amount of colloidal gold was observed. The growth of the particles from $4.7 \mathrm{~nm}$ to $5.1 \mathrm{~nm}$ (according to the SAXS-spectra) is in rough agreement with the increasing amount of colloidal gold. These observations are ascribed to a weak influence of the ligands in the densified coatings leading first to smaller particles compared to $1: 1$ and later to their growth with further densification probably due to diffusion and reduction of complexed $\mathrm{Au}$-ions surrounding the colloids. This process becomes important above $200^{\circ} \mathrm{C}$ as can be seen from Tables 1 and 2 taking into account that the total volume of colloidal gold is growing as $r^{3}$. The growth of the particles with densification might be the result of thermally activated diffusion and at least partially of a growing decomposition of the Au-stabilizer complex. This model is also valid in the case of Au:DIAMO $=1: 4$ (Fig. 5), where the stabilizer is able to control both, colloid size and the total amount of colloidal gold within the coatings considering the data given in Tables 1 and 2. First, small nuclei of reduced gold $(\sim 1 \mathrm{~nm})$ are detected which start to grow by diffusion and coalescence during thermal densification leading to particles with a size to $5 \mathrm{~nm}$. This particle growth has also been confirmed by HTEM-investigations which are presented in $[5,13]$. The size of the colloids as determined by electron microscopy is in agreement with the results given in Table 1 .

The effect of THIO (1:1) on the formation of the colloids needs a slightly modified interpretation. Up to $150^{\circ} \mathrm{C}$, THIO is able to suppress the formation of colloids completely (see Fig. 6 and Tables 1 and 2) despite the fact that a complexation of all $\mathrm{Au}$-ions by THIO should not be possible yet. At $200^{\circ} \mathrm{C}$ however, the size of the colloids has already reached $4.8 \mathrm{~nm}$. Thus it is supposed that to $150^{\circ} \mathrm{C}$ the ligands influence the redox-potential within the sol considerably preventing a reduction of gold ions. Beyond $150^{\circ} \mathrm{C}$ THIO then either starts to change its co-ordination of the gold-ions leading to an onset in the formation of $\mathrm{Au}$-nuclei or the reduction of $\mathrm{Au}$-ions is initialized by a thermal reduction of weakly stabilized gold. This description of the differences between DIAMO and THIO is in accordance with Pearon's concept of hard and weak acids and bases [6,21] where $\mathrm{Au}^{3+}$ belongs to the weak Lewis acids and should form more stable complexes with soft bases (i.e., thiosilanes) than with hard bases (aminosilanes). The con- 
tinuous decrease colloid size from about $4.8 \mathrm{~nm}$ to $4.2 \mathrm{~nm}$ as a function of temperature above $150^{\circ} \mathrm{C}$ is not understood yet within this model.

A general result from Figs. 3-6 is the fact that stabilization effects increase with higher DIAMO content and that THIO is a stronger stabilizer than DIAMO. Considering Table 1, it was also found that the colloids always reach about the same size $(\sim 5$ $\mathrm{nm})$ at $300^{\circ} \mathrm{C}$ independent of kind and ratio of the stabilizer in the sol. This might be a hint for a growth mechanism of the colloids up to $300^{\circ} \mathrm{C}$ which is dominated by thermally activated diffusion of Au-ions or atoms.

Slopes in the power law scattering range (Figs. 7-10) which strongly depend on densification temperature and stabilizer give an insight in the structure of the interface between matrix and core of the colloids. To interpret the experimental observations, there have be considered three cases of power law scattering with $I(q) \sim I(0) * q^{-\alpha}$ : Slopes around $\alpha$ $=4$, slopes smaller than 4 , and those significant higher than $\alpha=4$. A value of 4 is typical for dense particles with uniform electron density and a smooth and sharp phase boundary [22]. The case $\alpha>4$ is ascribed according to the literature [10,23-26] to a continuous decay in the electron density from the bulk material to the matrix. This is generally characterized as a 'fuzzy' surface $[24,10]$. The electronic density in the interface is written in this model as a convolution product of a theoretically sharp electronic density of the colloids and a smoothing function [27] which takes into account the width of the interface. If the smoothing function is assumed to be a Gaussian with standard deviation $\sigma$ (Fig. 11), the Porod law using the invariant $Q_{0}$ and the average intersect length $\langle l\rangle$ gets [10]

$\lim _{q \rightarrow \infty}\left\{\log \left[q^{4} I(q)\right]\right\}=-q^{2} \sigma^{2}+\log 8 \pi Q_{0} /\langle l\rangle$.

$\sigma$ which was experimentally determined from the SAXS spectra can be taken as an approximation of the mean thickness of the 'fuzzy' interface between colloid and matrix. The FWHM of a Gaussian is given by $2.36 \sigma$. Slopes between $\alpha=3$ to 4 , which are especially observed in the case of low densification temperatures here, are attributed to fractal surfaces $[8,24]$ with $I(q) \sim I(0) * q^{-(6-D)}$ where $D$ is the fractal dimension of the surface. An interpretation of the results within a model of mass fractals can be excluded as the colloids are generally spherical and mostly single crystalline in the core as confirmed by HRTEM and WAXS investigations.

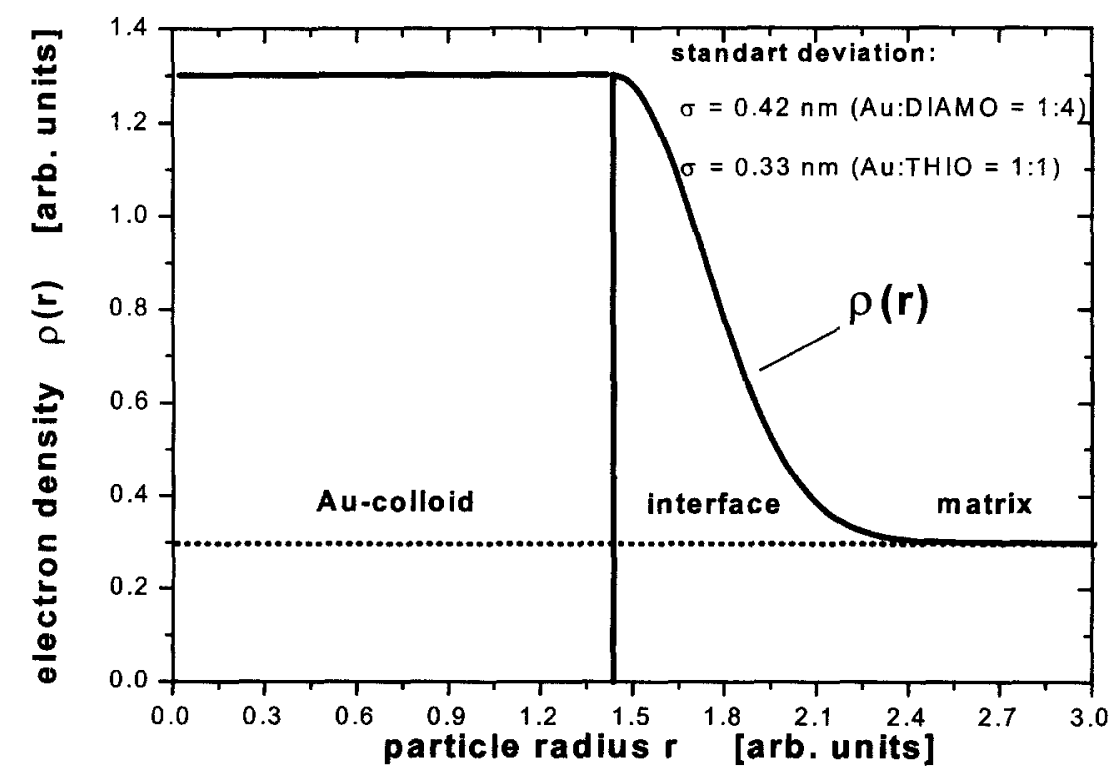

Fig. 11. Model of the electronic density $\rho(r)$ in the interface between matrix and colloids in the case of 'fuzzy' surfaces assuming a Gaussian with standard deviation $\sigma$ as smoothing function (estimated experimental error in $\sigma: \pm 0.12$ ). 
Deviations are found only at the surface of the colloids. In Fig. 7 no systematic variation of the slopes can be observed in accordance with the discussion of the values of $R_{\mathrm{g}}$ confirming the model of a spontaneous, fast and mostly uncontrolled formation of the colloids arising surfaces with a fractal dimension of about 2.4. Similar interpretations are proposed in the literature in the discussion of the reaction dimensions in catalysis of dispersed metals [28] and are also found in lignite coal [24], in porous glasses and glassy carbon [8].

An influence of densification temperature on the geometric structure of the surface of the colloids or a rearrangement within the interface was not detected. In the case of $\mathrm{Au}: \mathrm{DIAMO}=1: 2$, the observed slopes continuously increase from about 3 up to 4 . At $80^{\circ} \mathrm{C}$, the surface of the colloids has a lower fractal dimension than in Fig. 7 (Au:DIAMO =1:1). The geometry of the surface of the colloids is now strongly affected by the densification temperature. This indicates a growing densification of the surface of the colloids and formation of a sharp phase boundary at higher temperatures which might be due to a partial decomposition of DIAMO or its complex or due to a catalytic densification of the matrix surrounding the colloids by the functionalized silanes. In this picture, a higher stabilizer ratio first leads to a lower fractal dimension of the surface. Then, the proposed stabilizer changes at higher temperature finally lead at $\mathrm{Au}: \mathrm{DIAMO}=1: 2$ to a sharp phase boundary. This tendency is also observed for Au:DIAMO $=1: 4$ up to $150^{\circ} \mathrm{C}$. The lower temperature $\left(150^{\circ} \mathrm{C}\right.$ instead of $300^{\circ} \mathrm{C}$ ) to form a sharp boundary might be an effect of the higher amount of complexed gold ions surrounding the colloids leading to smaller diffusion lengths and thereby less thermal activation energy necessary for $\mathrm{Au}$ ions to reach the colloids. Above $150^{\circ} \mathrm{C}$ however, slopes which are significant greater than 4 are observed. To explain this, the model of a continuously decay in the electronic density in the interface as given in Fig. 11 is proposed leading to a mean width of the 'fuzzy' interface $\sigma$ of about $0.4-0.5 \mathrm{~nm}$. As DIAMO is known to act as a catalyst in the network formation of sol-gel systems [5], this 'fuzzy' interface is attributed to be the result of the formation of a dense, glass-like shell at the surface of the colloids where the stabilizer concentration is supposed to be higher than in the outer matrix (see also Fig. 2). This interpretation is supported by HRTEM investigations of similar sol-gel powders containing nanoscaled $\mathrm{Au}$ - or Ag-colloids (Au:DI$\mathrm{AMO}=1: 4, T$ up to $1000^{\circ} \mathrm{C}$ ) were often such a shell with a thickness up to several $1-3 \mathrm{~nm}$ surrounding the colloids could be observed [29]. It had been impossible to remove this shell from the surface of the colloids by ion-etching. A systematic investigation concerning the conditions of the formation of the shell and their detailed characterization has not been done. If the interface region only had a higher mechanic density than the matrix, this would not explain the observed slopes as its electronic density should be only slightly higher than in the matrix then, which is about one order of magnitude lower than the electronic density of gold. Thus, it is concluded that a high stabilizer ratio at the surface of the colloids acts in a first step to densify the interface region stronger than the outer matrix. This densified shell effects then as a diffusion barrier for further Au-ions or atoms surrounding the colloids leading to a slower growth of the particles at higher densification temperature and to an enrichment of Au-ions or atoms within or at the interface which normally could join the colloids by diffusion.

This model must be checked in more detail by further investigations at higher densification temperatures, but it also offers an access to the interpretation of the broad UV-VIS spectra (see Figs. 5 and 6) and to the extraordinary non-linear optical properties of the Au-colloids which are also strongly affected by the stabilizer [6]. Both observations should be strongly influenced by changed electronic or dielectric properties of the interface region compared to bulk gold for example.

A first HRTEM picture taken at the sample densified at $300^{\circ} \mathrm{C}$ is presented in Fig. 12 showing an isolated, single-crystalline Au-colloid embedded in the sol-gel matrix. It is clearly visible that the contrast at the surface of the colloid is different from the surrounding matrix indicating the formation of a shell-like structure with a thickness of about 0.5 to $0.8 \mathrm{~nm}$ with a decreasing electronic density. Such a shell structure is not visible in the case of colloids densified at $150^{\circ} \mathrm{C}$ (Au:DIAMO $=1: 4$, same preparation) [13]. The observation of a continuous interface between matrix and Au-colloid supports the interpretation of the SAXS data and the model given above. 


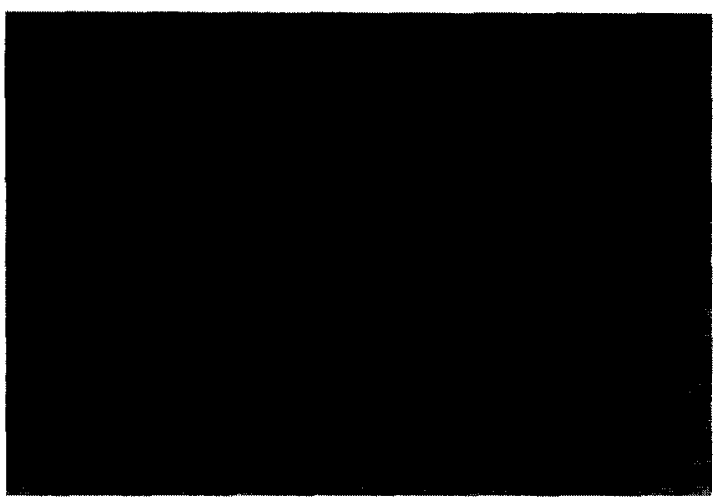

Fig. 12. HRTEM picture a nanoscaled single crystalline Au-colloid $(r=\sim 4 \mathrm{~nm})$ embedded in the glass-like sol-gel matrix, densified at $300^{\circ} \mathrm{C}$ (Au:DIAMO $=1: 4$ ).

It also confirms the result of the calculation of the thickness of the interface from the SAXS spectra.

In the case of THIO (1:1), the elaborated model can also be applied. Up to $150^{\circ} \mathrm{C}$, no colloids are found in the SAXS-spectra (Fig. 10) due to strong complexation. Then, starting at $200^{\circ} \mathrm{C}$, a clear scattering signal of the colloids is detected. The formation of the colloids is not spontaneous now because in this case, as an evolution as in Fig. 7 should be expected. Here however, a sharp interface is found first which is probably due to the formation of nuclei and their growth by diffusion of complexed Au-ions surrounding the colloids (comparable to Fig. 8, $200^{\circ} \mathrm{C}-300^{\circ} \mathrm{C}$ ). At $300^{\circ} \mathrm{C}$ finally, the same evolution as found at DIAMO $\left(1: 4,200^{\circ} \mathrm{C}-300^{\circ} \mathrm{C}\right)$ is observed. It is interpreted again as a consequence of the densification of the network surrounding the colloids probably acting as a diffusion barrier for further $\mathrm{Au}$-ions or atoms. The mean broadness of the interface is only about $\sigma=0.33 \mathrm{~nm}$ now, which might be connected with the lower coverage of the surface by the stabilizer.

\section{Conclusions}

The size of colloids can be controlled by the densification temperature and stabilizer concentration used in the sol. In the Porod region of the SAXS spectra, slopes which differ significantly from -4 were attributed to the interface between matrix and colloids whose electronic structure changes strongly with the preparation parameters. These results offer an access to explain the observed deviations of the experimental UV-VIS spectra from theoretical calculations and the non-linear optical properties of the colloids in the matrix, which are considerably higher than in molten glasses and also depend strongly on the stabilizers.

\section{Acknowledgements}

This work was financially supported by the State of Saarland and the Commission of the European Community under the COMETT program with participation of EUROMATERIAUX. The authors also thank Dr U. Werner for the HRTEM investigations.

\section{References}

[1] A. Naudon, Nato ASI Series, Ser. C, Vol. 451, 1995, pp. 203-220.

[2] G. Materlik, C.J. Sparks, K. Fischer, eds., Resonant Anomalous X-Ray Scattering (North-Holland, Amsterdam, 1994).

[3] D. Schulze, ed., Physical Research, Vol. 12, Amorphous Structures - Methods and Results (Akademie, Berlin, 1990).

[4] L. Spanhel, M. Mennig, H. Schmidt, Bol. Soc. Esp. Ceram. VID 31-C (7) (1992) 612.

[5] M. Schmitt, PhD thesis, Universität des Saarlandes (1997), to be published.

[6] M. Mennig, U. Becker, G. Jung, H. Schmidt, Sol-Gel-Opt. III, SPIE 2288 (1994) 130.

[7] V. Vezin, P. Goudeau, A. Naudon, A. Halimaoui, G. Bomchil, Appl. Phys, Lett. 60 (21) (1992) 2625.

[8] P. Fratzl, G. Vogl, S. Klaumünzer, J. Appl. Crystallogr. 24 (1991) 588.

[9] A. Emmerling, R. Gerlach, R. Goswin, J. Gross, G. Reichenauer, J. Fricke, H.-G. Haubold, J. Appl. Crystallogr. 24 (1991) 781.

[10] A. Naudon, P. Goudeau, A. Halimaoui, B. Lambert, G. Bomchil, J. Appl. Phys. 75 (2) (1994) 780.

[11] A. Naudon, T. Slimani, P. Goudeau, J. Appl. Crystallogr. 24 (1991) 501

[12] T. Slimani, P. Goudeau, A. Naudon, G. Farges, J.L. Derep, J. Appl. Crystallogr. 24 (1991) 638.

[13] B. Kutsch, O. Lyon, M. Schmitt, M. Mennig, H. Schmidt, submitted to J. Appl. Crystallogr.

[14] J.M. Dubuisson, J.M. Dauvergne, C. Depautex, P. Vachette, C.E. Williams, Nucl. Instrum. Methods A246 (1986) 636.

[15] M. Mennig, U. Becker, M. Schmitt, H. Schmidt, Proc. 8th CIMTEC, Florence, Italy, 1994.

[16] G. Mie, Ann. Phys. 25 (1908) 377.

[17] B. Dusemund, MSc thesis, Universität des Saarlandes, Saarbrücken (1991). 
[25] P.W. Schmidt, D. Avnir, D. Levy, A. Höhr, M. Steiner, A. Röll, J. Chem. Phys. 94 (2) (1991) 1474.

[18] H. Hövel, S. Fritz, A. Hilger, U. Kreibig, M. Vollmer, Phys. Rev. B48 (24) (1993) 18178.

[19] B.N.J. Perrson, Surf. Sci. 281 (1993) 153.

[20] E. Riedel, Anorganische Chemie, 2nd Ed. (Walter de Gruyter, Berlin, 1990).

[21] Pearson, J. Am. Chem. Soc. 85 (1963) 3533.

[22] A. Guinier, G. Fournet, Small Angle Scattering of X-Rays (Wiley, New York, 1955).

[23] P. Wong, A. Bray, J. Appl. Crystallogr. 21 (1988) 786.

[24] P. Schmidt, J. Appl. Crystallogr. 21 (1988) 786.
[26] S. Cicciarello, J. Goodisman, H. Brumberger, J. Appl. Crystallogr. 21 (1988) 117

[27] J.T. Koberstein, B. Morra, R.S. Stein, J. Appl. Crystallogr. 13 (1980) 34.

[28] D. Farin, D. Avnir, J. Am. Chem. Soc. 110 (1988) 2039.

[29] U. Werner, Institut für Neue Materialien, Saarbrücken, Germany, unpublished results and private communication 